\title{
Random-matrix theory of quantum size effects on nuclear magnetic resonance in metal particles
}

\author{
C. W. J. Beenakker \\ Instituut-Lorentz, University of Leiden, P.O. Box 9506, 2300 RA Leiden, The Netherlands
}

(Received 19 August 1994)

\begin{abstract}
The distribution function of the local density of states is computed exactly for the Wigner-Dyson ensemble of random Hamiltonians. In the absence of time-reversal symmetry, precise agreement is obtained with the supersymmetry theory by Efetov and Prigodin of the NMR line shape in disordered metal particles. Upon breaking time-reversal symmetry, the variance of the Knight shift in the smallest particles is reduced by a universal factor of $2 / 3$.
\end{abstract}

A new quantum size effect in small metal particles has been predicted by Efetov and Prigodin. ${ }^{1}$ They computed the spectral line shape for nuclear magnetic resonance (NMR) and found that the resonance becomes very broad upon decreasing the temperature and particle size, due to large fluctuations in the Knight shift at different points in the sample. Similar results for a disordered wire have been reported by Mirlin and Fyodorov, ${ }^{2}$ who extended earlier work on one-dimensional conductors. ${ }^{3-6}$ Recent NMR spectroscopy on a monodisperse array of nanometer-size $\mathrm{Pt}$ particles is in striking agreement with the theory. ${ }^{7}$ The essential difference with quantum size effects on thermodynamic properties ${ }^{8}$ is that NMR in a metal measures the local density of states $\rho(E, \mathbf{r})=$ $\sum_{n} \delta\left(E-E_{n}\right)\left|\Psi_{n}(\mathbf{r})\right|^{2}$, and thus depends both on the energy levels $E_{n}$ and the wave functions $\Psi_{n}(\mathbf{r})$ of the valence electrons. The sensitivity to the local density of states occurs because the frequency of microwave absorption by a nucleus is shifted as a result of the Fermi contact interaction between the nuclear spin and the electron spin. (This is known as the Knight shift. ${ }^{9}$ ) The frequency shift $\delta \omega_{i}$ for a nucleus at position $\mathbf{r}_{i}$ is linearly proportional to $\rho\left(E_{F}, \mathbf{r}_{i}\right)$ (with $E_{F}$ the Fermi energy). Different nuclei have different frequency shifts, which is observed as a broadening of the resonance in an ensemble of particles. To determine the broadening one has to consider the combined effect of particle-to-particle fluctuations in the energy spectrum and spatial fluctuations of the wave functions within the disordered particles. The strong spectral correlations make the theory considerably more complex than for an ideal spherical particle. ${ }^{10,11}$

Efetov and Prigodin computed the fluctuations in the local density of states from a microscopic model of a disordered metal particle with elastic impurity scattering in a strong magnetic field by mapping it onto a supersymmetric nonlinear $\sigma$ model. The traditional approach $^{8}$ to quantum size effects in metal particles is based on random-matrix theory. Following Gor'kov and Eliashberg, ${ }^{12}$ it is assumed that the Hamiltonian $\mathcal{H}$ of an irregularly shaped metal particle is a random $N \times N$ Hermitian matrix, with the Gaussian distribution

$$
P(\mathcal{H})=C \exp \left(-c \operatorname{Tr} \mathcal{H}^{2}\right)
$$

originally introduced by Wigner and Dyson for the spectrum of a heavy nucleus. ${ }^{13}$ The coefficient $c$ determines the mean level spacing $\Delta$ (which in the limit $N \rightarrow \infty$ is uniform in the bulk of the spectrum), and $C$ is a normalization constant. In the past, random-matrix theory has been applied to quantum size effects on thermodynamic properties of metal particles, ${ }^{8,14}$ in agreement with microscopic theories. ${ }^{15-17}$ These applications involve the distribution of the set of eigenvalues $\left\{E_{n}\right\}$ of $\mathcal{H}$, which follows from $P(\mathcal{H})$ on integrating out the eigenvectors $\left\{\Psi_{n}\right\}$. In contrast, the NMR line shape depends on the joint distribution of the $E$ 's and $\Psi$ 's. Problems of this type have not previously been tackled by random-matrix theory.

It is the purpose of this paper to show how the anomalous broadening of the NMR line shape can be obtained directly from the Wigner-Dyson distribution (1), without any further assumption. In the absence of time-reversal symmetry we recover precisely the results of Ref. 1. Experiments on nanometer-size particles are typically performed in the presence of time-reversal symmetry. (The authors of Ref. 7 estimate that to break time-reversal symmetry in their system would require magnetic fields of the order of $1000 \mathrm{~T}$, two orders of magnitude greater than the experimental fields.) Random-matrix theory is particularly suited to investigate the dependence of the fluctuations on fundamental symmetries of the Hamiltonian. A celebrated example is the reduction by a factor of $1 / 2$ of the variance of the universal conductance fluctuations, upon breaking time-reversal symmetry. ${ }^{17-19} \mathrm{We}$ will show that the variance of the Knight shift has a different reduction factor of $2 / 3$, provided the level spacing is much greater than both the level broadening and the temperature.

Let us first reformulate the problem of the NMR line shape in the framework of random-matrix theory. The intensity $I(\omega)$ of the resonance at frequency $\omega$ is given by the distribution $P(\rho)$ of the local density of states upon rescaling,

$$
I(\omega)=a P\left(\rho=b\left(\omega-\omega_{0}\right)\right)
$$

with microscopic parameters $a, b, \omega_{0}$. The distribution 
$P(\rho)$ is defined by

$$
P(\rho)=\left\langle\delta\left(\rho-\rho\left(E_{F}, \mathbf{r}\right)\right)\right\rangle,
$$

where the average \langle\rangle is a spatial average over the total volume occupied by the particles. The local density of states $\rho(E, \mathbf{r})$ is given by

$$
\begin{aligned}
& \rho(E, \mathbf{r})=\sum_{n} f\left(E-E_{n}\right)\left|\Psi_{n}(\mathbf{r})\right|^{2}, \\
& f(E)=(\gamma / 2 \pi)\left(E^{2}+\frac{1}{4} \gamma^{2}\right)^{-1}
\end{aligned}
$$

where $\gamma$ is the broadening of the levels due to tunneling into the medium in which the particles are imbedded. Equations (2) and (3) assume that $\gamma$ is greater than the temperature, the electronic Zeeman energy, and the spinorbit scattering rate. For completely isolated particles, even-odd electron number effects, which are not considered here, play a role. ${ }^{8}$ (These effects are expected to be relatively unimportant in the metal-cluster compounds of current experimental interest. ${ }^{7}$ )

For an $N$-dimensional Hamiltonian $\mathcal{H}$, the continuous variable $\mathbf{r}$ is replaced by the index $m=1,2, \ldots N$, and $\left|\Psi_{n}(\mathbf{r})\right|^{2}$ becomes $(N / V)\left|U_{m n}\right|^{2}$, with $U$ the unitary matrix that diagonalizes $\mathcal{H}$ and $V$ the volume of a particle. In the absence of time-reversal symmetry, $U$ varies over the full unitary group. This is relevant to NMR for very strong magnetic fields and not too small particles, and is the case considered in Ref. 1. If the flux penetrating a particle is much less ${ }^{20}$ than $h / e$, then time-reversal symmetry is not broken and $U$ is restricted to the orthogonal group. The orthogonal and unitary ensembles are characterized by the index $\beta=1,2$, which counts how many real numbers $u_{m n, q}(q=1, \ldots \beta)$ define the matrix element $U_{m n}$. There exists a third symmetry class, characterized by $\beta=4$ and $U$ a symplectic matrix, which describes systems with time-reversal symmetry in the presence of strong spin-orbit scattering. ${ }^{8,13}$ All our $\beta$-dependent formulas for the local density of states apply also to the symplectic ensemble; however, the application to NMR requires a modification of the theory because spin and charge density are no longer directly related.

For each of the random-matrix ensembles, the average in Eq. (3) can be written as an integration over eigenvalues and eigenvectors, ${ }^{21}$

$$
\begin{aligned}
P(\rho)= & \int d E_{1} \cdots \int d E_{N} \int d U C \exp \left(-c \sum_{n} E_{n}^{2}\right) \\
& \times \prod_{i<j}\left|E_{i}-E_{j}\right|^{\beta} \\
& \times \delta\left(\rho-\frac{N}{V} \sum_{n} f\left(E_{n}\right)\left|U_{m n}\right|^{2}\right) .
\end{aligned}
$$

Here, and in what follows, we choose $E_{F}$ as the zero of energy. The Jacobian $\prod_{i<j}\left|E_{i}-E_{j}\right|^{\beta}$ introduces $\beta$ dependent correlations between the eigenvalues, in the form of level repulsion. The eigenvectors are uncorrelated with the eigenvalues, and distributed uniformly with measure $d U$.

The variance of the Knight shift requires the first two moments of $P(\rho)$. The general formula for the $p$ th moment is

$$
\overline{\rho^{p}}=(N / V)^{p}\left\langle\left(\sum_{n} f\left(E_{n}\right)\left|U_{m n}\right|^{2}\right)^{p}\right\rangle .
$$

The first moment evaluates trivially to

$$
\bar{\rho}=(V \Delta)^{-1} \equiv \rho_{0} .
$$

To evaluate the second moment we use the formula ${ }^{22}$

$$
\left\langle\left|U_{m n}\right|^{2}\left|U_{m n^{\prime}}\right|^{2}\right\rangle=\frac{\beta+2 \delta_{n n^{\prime}}}{N(\beta N+2)} .
$$

In the limit $N \rightarrow \infty$, at constant $V$ and $\Delta$, we find

$$
\begin{aligned}
\overline{\rho^{2}} / \rho_{0}^{2}= & 1+(1+2 / \beta) \Delta \int_{-\infty}^{\infty} d E f^{2}(E) \\
& -\Delta^{2} \int_{-\infty}^{\infty} d E \int_{-\infty}^{\infty} d E^{\prime} f(E) f\left(E^{\prime}\right) T_{2}\left(E-E^{\prime}\right) .
\end{aligned}
$$

The two-level cluster function

$$
T_{2}\left(E-E^{\prime}\right)=\Delta^{-2}-\left\langle\sum_{i \neq j} \delta\left(E-E_{i}\right) \delta\left(E^{\prime}-E_{j}\right)\right\rangle
$$

is known. ${ }^{21}$ For $\beta=2$ one has

$$
T_{2}(E)=(\pi E)^{-2} \sin ^{2}(\pi E / \Delta) .
$$

The expressions for $\beta=1,4$ are a little more complicated. ${ }^{21}$ The asymptotic behavior of Eq. (10) for $\Delta \gg \gamma$ is obtained from $\lim _{E \rightarrow 0} \Delta^{2} T_{2}(E)=1$; hence

$$
\overline{\rho^{2}} / \rho_{0}^{2}=(1+2 / \beta) \Delta \int_{-\infty}^{\infty} d E f^{2}(E)+O(\gamma / \Delta) .
$$

In the opposite regime, $\Delta \ll \gamma$, one may approximate $\Delta T_{2}(E) \simeq \delta(E)$; hence

$$
\overline{\rho^{2}} / \rho_{0}^{2}=1+\frac{2 \Delta}{\beta} \int_{-\infty}^{\infty} d E f^{2}(E)+O(\Delta / \gamma)^{2}
$$

From Eq. (10) one readily computes the variance $\operatorname{Var} K / \bar{K}=\overline{\rho^{2}} / \rho_{0}^{2}-1$ of the Knight shift. The result is plotted in Fig. 1 for $\beta=1,2$ (solid curves). The smalland large- $\Delta$ asymptotes (dashed curves) are both linear, but with different slopes:

$$
\operatorname{Var} K / \bar{K}=\frac{2 \Delta}{\pi \gamma} \times\left\{\begin{array}{ccc}
\frac{1}{\beta} & \text { if } \Delta \ll \gamma \\
\frac{2+\beta}{2 \beta} & \text { if } \Delta \gg \gamma
\end{array}\right.
$$

We have checked that the values for $\beta=2$ agree with Ref. 1. The transition $\beta=1 \rightarrow \beta=2$ on increasing the magnetic field is signaled by a reduction of $\operatorname{Var} K / \bar{K}$ by a factor of $2 / 3$ for $\Delta \gg \gamma$ and $1 / 2$ for $\Delta \ll \gamma$. The reduction by $1 / 2$ is the same as for the variance of the universal conductance fluctuations (UCF) in mesoscopic metals. In that case the broadening of the levels is always much greater than the level spacing. (Their ratio is the 


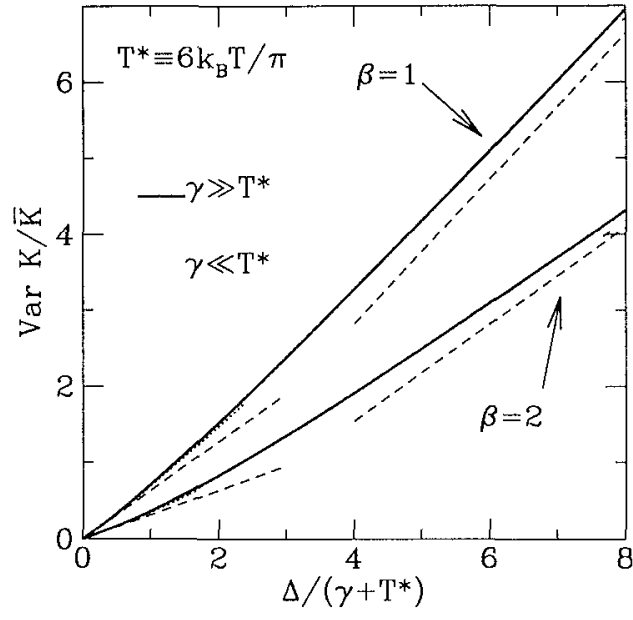

FIG. 1. Dependence of the variance of the Knight shift $K$ (normalized by its average $\bar{K}$ ) on the level spacing $\Delta$, with and without time-reversal symmetry $(\beta=1$ and 2$)$. The solid curves are computed from Eq. (10) for the case that the level broadening $\gamma$ is much greater than the thermal energy $k_{B} T$. The dotted curves are for the opposite case, $\gamma \ll k_{B} T$. The dashed lines are the asymptotes (13) and (14) for small and large $\Delta$. (The factor $6 / \pi$ in the definition of $T^{*}$ is chosen such that the solid and dotted curves have the same asymptotes.) Breaking of time-reversal symmetry reduces $\operatorname{Var} K$ by a factor of $1 / 2$ and $2 / 3$ for small and large $\Delta$, respectively. These factors are universal, independent of the relative magnitude of $\gamma$ and $k_{B} T$.

conductance in units of $e^{2} / h$, which is $\gg 1$ in a metal.) The reduction by $2 / 3$ has no analog for UCF.

So far we have assumed that the level broadening $\gamma$ is much greater than the thermal energy $k_{B} T$. At higher temperatures, the function $f(E)$ in Eq. (10) is to be replaced by the convolution $f_{T}(E)$ of the Lorentzian (5) with the derivative of the Fermi function. Its Fourier transform $f_{T}(k)=\int d E e^{\imath k E} f_{T}(E)$ is

$$
f_{T}(k)=\pi k_{B} T k\left[\exp \left(\frac{1}{2} \gamma|k|\right) \sinh \left(\pi k_{B} T k\right)\right]^{-1} .
$$

The variance of the Knight shift for $\gamma \ll k_{B} T$ is plotted also in Fig. 1 (dotted curves). The asymptotic formulas for small and large $\Delta$ become

$\operatorname{Var} K / \bar{K}=\frac{\Delta}{k_{B} T} \Phi\left(\frac{\gamma}{k_{B} T}\right) \times\left\{\begin{array}{cl}\frac{1}{\beta} & \text { if } \Delta \ll \gamma+k_{B} T \\ \frac{2+\beta}{2 \beta} & \text { if } \Delta \gg \gamma+k_{B} T\end{array}\right.$

$\Phi(s)=2 \pi \int_{0}^{\infty} d q e^{-q s} q^{2}(\sinh \pi q)^{-2}$.

We conclude that the reduction factor associated with $\beta=1 \rightarrow \beta=2$ is universal, independent of the relative magnitude of temperature and level broadening. This is relevant for experiments, which are typically in the regime that $k_{B} T$ and $\gamma$ are of comparable magnitude. ${ }^{7}$

We now turn to the complete distribution $P(\rho)$, given by Eq. (6). It is convenient to work with the (dimensionless) Laplace transform

$$
F(s)=\int_{0}^{\infty} d \rho \exp \left(-s \rho / \rho_{0}\right) P(\rho)
$$

and recover $P(\rho)$ at the end by inverting the transform. First, we average over the eigenvectors. It is known ${ }^{13,23}$ that, to leading order in $1 / N$, the $\beta N$ components $u_{m n, q}$ $(n=1,2, \ldots N ; q=1, \ldots \beta)$ of a single row of $U$ are independently distributed Gaussian variables with zero mean and variance $1 / \beta N$. Carrying out the Gaussian integrations, we find

$$
\begin{aligned}
& F(s)=\left\langle\prod_{n=1}^{N}\left(1-g\left(E_{n}, s\right)\right)\right\rangle \\
& g(E, s)=1-[1+(2 s \Delta / \beta) f(E)]^{-\beta / 2} .
\end{aligned}
$$

The remaining average over the eigenvalues can be carried out using the method of orthogonal polynomials. ${ }^{21}$ This method works for any $\beta$, but is simplest for the case $\beta=2$. In that case the function $g$ is a Lorentzian in $E$,

$$
g(E, s)=(s \gamma \Delta / 2 \pi)\left(E^{2}+\frac{1}{4} \Gamma^{2}\right)^{-1},
$$

with $\Gamma \equiv \gamma(1+2 s \Delta / \pi \gamma)^{1 / 2}$. The large- $N$ limit of Eq. (19) for $\beta=2$ is given by the Fredholm determinant

$$
F(s)=\prod_{n=1}^{\infty}\left(1-\lambda_{n}(s)\right),
$$

where $\lambda_{n}$ is an eigenvalue of the integral equation

$$
\int_{-\infty}^{\infty} d E^{\prime} g\left(E^{\prime}, s\right) T_{2}^{1 / 2}\left(E-E^{\prime}\right) \phi\left(E^{\prime}\right)=\lambda \phi(E) .
$$

Fourier transformation gives

$$
\begin{aligned}
& \frac{1}{2 \pi} \int_{-\pi / \Delta}^{\pi / \Delta} d k^{\prime} g\left(k-k^{\prime}, s\right) \phi\left(k^{\prime}\right)=\lambda \phi(k), \\
& g(k, s)=(s \gamma \Delta / \Gamma) \exp \left(-\frac{1}{2} \Gamma|k|\right) .
\end{aligned}
$$

To evaluate the Fredholm determinant of Eq. (24), we discretize $k \in(-\pi / \Delta, \pi / \Delta)$ as $k_{n}=(\pi / \Delta)(-1+2 n / M)$, $n=1,2, \ldots M$, and then take the limit $M \rightarrow \infty:^{24}$

$$
\begin{aligned}
F(s) & =\lim _{M \rightarrow \infty} \operatorname{Det}\left[\delta_{n m}-\frac{s \gamma}{M \Gamma} \exp \left(-\frac{\pi \Gamma|n-m|}{M \Delta}\right)\right] \\
& =e^{-\alpha \Gamma / \gamma}\left(\cosh \alpha+\frac{1}{2}(\gamma / \Gamma+\Gamma / \gamma) \sinh \alpha\right) .
\end{aligned}
$$

Inversion of the Laplace transform yields finally

$$
\begin{aligned}
P(\rho)= & \rho_{0}^{-1}(\alpha / 2 \pi)^{1 / 2} x^{-3 / 2} \exp \left[-\frac{1}{2} \alpha\left(x+x^{-1}\right)\right] \\
& \times\left(\cosh \alpha+\frac{1}{2}\left(x+x^{-1}-\alpha^{-1}\right) \sinh \alpha\right) .
\end{aligned}
$$

Here $x \equiv \rho / \rho_{0}$ and $\alpha \equiv \pi \gamma / \Delta$. Equation (27) is precisely the distribution of Efetov and Prigodin. ${ }^{1}$

This solves completely the problem for $\beta=2$ and zero temperature. For $\beta=1,4$ and $T \neq 0$ the distribution function can still be written as a Fredholm determinant, which then has to be evaluated numerically. The WignerDyson distribution (1) can only describe the pure symmetry classes $(\beta=1,2$, or 4$)$. The transition between symmetry classes might be studied by means of an extension known as Dyson's Brownian motion model. ${ }^{21} \mathrm{We}$ leave these problems for future work.

In summary, we have derived the result of Efetov and Prigodin ${ }^{1}$ for the NMR line shape in the absence of time- 
reversal symmetry from the single assumption that the Hamiltonian of the particle is a member of the WignerDyson ensemble of random-matrix theory. A 2/3 reduction of the variance of the Knight shift in the smallest particles has been predicted to occur upon breaking timereversal symmetry.
I am indebted to K. B. Efetov for suggesting this problem as a challenge for random-matrix theory. B. Rejaei helped me to compute the determinant in Eq. (26). Discussions with $\mathrm{H}$. B. Brom on the experimental aspects have been most helpful. This work was supported by the Dutch Science Foundation NWO/FOM.
${ }^{1}$ K. B. Efetov and V. N. Prigodin, Phys. Rev. Lett. 70, 1315 (1993); Mod. Phys. Lett. B 7, 981 (1993).

${ }^{2}$ A. D. Mirlin and Y. V. Fyodorov, Europhys. Lett. 25, 669 (1994).

${ }^{3}$ I. Ya. Yanchev, Fiz. Tekh. Poluprovodn. 8, 1494 (1974) [Sov. Phys. Semicond. 8, 973 (1975)].

${ }^{4}$ V. V. Avilov, L. N. Bulaevskiü, and O. N. Dorokhov, Pis'ma Zh. Eksp. Teor. Fiz. 42, 156 (1985) [JETP Lett. 42, 192 (1985)].

${ }^{5}$ B. L. Al'tshuler and V. N. Prigodin, Zh. Eksp. Teor. Fiz. 95, 348 (1989) [Sov. Phys. JETP 68, 198 (1989)].

${ }^{6}$ A. Vainrub, S. Vija, E. Lippmaa, V. Prigodin, R. Beha, and M. Mehring, Phys. Rev. Lett. 69, 3116 (1992); V. N. Prigodin, Phys. Rev. B 47, 10885 (1993).

${ }^{7}$ H. B. Brom, F. C. Fritschij, D. van der Putten, F. A. Hanneman, L. J. de Jongh, and G. Schmid (unpublished).

${ }^{8}$ W. P. Halperin, Rev. Mod. Phys. 58, 533 (1986).

${ }^{9}$ C. P. Slichter, Principles of Magnetic Resonance (Springer, New York, 1980).

${ }^{10}$ I. Yu, A. A. V. Gibson, E. R. Hunt, and W. P. Halperin, Phys. Rev. Lett. 44, 348 (1980).

${ }^{11}$ C. D. Makowka, C. P. Slichter, and J. H. Sinfelt, Phys. Rev. B 31, 5663 (1985).

${ }^{12}$ L. P. Gor'kov and G. M. Eliashberg, Zh. Eksp. Teor. Fiz. 48, 1407 (1965) [Sov. Phys. JETP 21, 940 (1965)].

${ }^{13}$ T. A. Brody, J. Flores, J. B. French, P. A. Mello, A. Pandey, and S. S. M. Wong, Rev. Mod. Phys. 53, 385 (1981).
${ }^{14}$ R. Denton, B. Mühlschlegel, and D. J. Scalapino, Phys. Rev. Lett. 26, 707 (1971); Phys. Rev. B 7, 3589 (1973).

${ }^{15}$ K. B. Efetov, Adv. Phys. 32, 53 (1983).

${ }^{16}$ J. J. M. Verbaarschot, H. A. Weidenmüller, and M. R. Zirnbauer, Phys. Rep. 129, 367 (1985).

${ }^{17}$ B. L. Al'tshuler and B. I. Shklovskiĭ, Zh. Eksp. Teor. Fiz. 91, 220 (1986) [Sov. Phys. JETP 64, 127 (1986)].

${ }^{18}$ A. D. Stone, P. A. Mello, K. A. Muttalib, and J.-L. Pichard, in Mesoscopic Phenomena in Solids, edited by B. L. Al'tshuler, P. A. Lee, and R. A. Webb (North-Holland, Amsterdam, 1991).

${ }^{19}$ C. W. J. Beenakker, Phys. Rev. Lett. 70, 1155 (1993).

${ }^{20}$ Time-reversal symmetry is effectively broken when the flux through the particle exceeds $(h / e) g^{-1 / 2}$, where $g$ is its conductance (in units of $e^{2} / h$ ). Cf. N. Dupuis and G. Montambaux, Phys. Rev. B 43, 14390 (1991).

${ }^{21}$ M. L. Mehta, Random Matrices (Academic, New York, 1991).

22 N. Ullah, Nucl. Phys. 58, 65 (1964).

${ }^{23}$ P. Pereyra and P. A. Mello, J. Phys. A 16, 237 (1983).

${ }^{24}$ The determinant in Eq. (26) is evaluated as follows: Let $D_{M}$ be the determinant of the $M \times M$ matrix with elements $\delta_{n m}+a b^{|n-m|}$. By adding rows and columns the matrix can be brought to a tridiagonal form, which leads to the recursion relation $D_{M}=\left(1+a+(1-a) b^{2}\right) D_{M-1}-b^{2} D_{M-2}$. Equation (26) follows in the limit $M \rightarrow \infty$. 